\title{
Silicones with enhanced permittivity for dielectric elastomer actuators
}

\author{
Simon J. Dünki ${ }^{\mathrm{a}, \mathrm{b}}$, Mihaela Dascalu ${ }^{\mathrm{a}}$, Frank A. Nüesch ${ }^{\mathrm{a}, \mathrm{b}}$, Dorina M. Opris ${ }^{\mathrm{a}^{*}}$ \\ ${ }^{a}$ Laboratory for Functional Polymers, Überlandstr. 129, CH-8600, Dübendorf, Switzerland; \\ ${ }^{b}$ École Polytechnique Fédérale de Lausanne (EPFL), Institut des Matériaux, Station 12, CH 1015, \\ Lausanne, Switzerland
}

\begin{abstract}
The research efforts for silicone based elastomers with high dielectric permittivity $\left(\varepsilon^{\prime}\right)$ intensified significantly in the last years since such materials would allow the construction of dielectric elastomer actuators (DEA) with low operation voltages. Polar groups can be introduced to elastomers to adjust their permittivity. The results obtained regarding the functionalization of silicones with polar nitrile $(\mathrm{CN})$ and trifluoropropyl $\left(\mathrm{CF}_{3}\right)$ groups are presented. Those with $\mathrm{CN}$ groups were synthesized via anionic polymerization of nitrile containing cyclosiloxanes or via a post-polymerization modification of functional polysiloxanes. Polysiloxanes containing $\mathrm{CF}_{3}$ groups were prepared by anionic copolymerization of 1,3,5-tris(3,3,3-trifluoropropyl)-1,3,5-trimethylcyclosiloxane with octamethylcyclotetrasiloxane. Importantly, we have found that all polysiloxanes have glass transition temperatures $\left(T_{g}\right)$ well below room temperature $\left(<-50{ }^{\circ} \mathrm{C}\right)$. This ensures that the materials turn into true elastomers after cross-linking. In addition to this, a linear increase in $\varepsilon^{\prime}$ with increasing content of polar groups was observed with maximum values of $\varepsilon^{\prime}=18$ and $\varepsilon^{\prime}=8.8$ for polysiloxanes modified at every repeating unit with either $\mathrm{CN}_{\text {or }} \mathrm{CF}_{3}$ groups, respectively.
\end{abstract}

Keywords: dielectric elastomer actuators, polar silicones, dipoles, thiol-ene addition, high permittivity elastomers

\section{INTRODUCTION}

Dielectric elastomer actuators consist of two stretchable electrodes that sandwich a thin elastomer film. When a voltage is applied, due to Coulombic attraction forces between opposite charges on the electrodes, the elastomers contract in thickness and mechanical work is generated from electrical energy. ${ }^{1}$

Pelrine et al. introduced a simple model for the actuation strain in $\mathrm{z}$ direction $\mathrm{s}_{\mathrm{z}}{ }^{2}$

$$
S_{z}=\frac{\varepsilon^{\prime} \varepsilon_{0} E^{2}}{Y}=\frac{\varepsilon^{\prime} \varepsilon_{0}}{Y}\left(\frac{U}{d}\right)^{2}
$$

where $\varepsilon^{\prime}$ is the permittivity and $Y$ is the Young's modulus, $\varepsilon_{0}=8.854 \times 10^{-12} \mathrm{~F} \cdot \mathrm{m}^{-1}$ is the vacuum permittivity, $d$ is the thickness of the elastomer, and $U$ is the applied voltage.

A wide range of elastomers, including silicones, polyurethane, and acrylic polymers like the commercial VHB elastomer have been evaluated as dielectric material, but their low dielectric permittivity does not allow construction of DEA operated at low voltage which hinders many interesting potential applications.

Silicones are advantageous over other materials regarding properties such as low conductivity, low viscoelastic losses, and stable mechanical properties over a wide temperature and frequency range. It is therefore not surprising that silicone based elastomers made it into the first commercial products. An overview of materials used for DEA is given in a book edited by Carpi et al. as well as in recent reviews, ${ }^{3-5}$ while an overview of materials that were developed in our lab was given recently. ${ }^{6}$

*dorina.opris@empa.ch: phone 004158765 4304; fax 004158765 1122; empa.ch

Electroactive Polymer Actuators and Devices (EAPAD) 2016, edited by Yoseph Bar-Cohen, Proc. of SPIE Vol. 9798, 97982K · @ 2016 SPIE · CCC code: 0277-786X/16/\$18 · doi: 10.1117/12.2218881 
The main drawback of silicones is their low permittivity $\varepsilon$ ', which is typically below 3 . Therefore significant efforts have been invested to improve on this aspect.

Several strategies have been used to increase the permittivity of silicones by blending them with high permittivity fillers or by modifying them with polar groups. As high permittivity fillers either ceramic particles such as $\mathrm{BaTiO}_{3}{ }^{7} \mathrm{TiO}_{2}{ }^{8}$ $\mathrm{CaCuTi}_{4} \mathrm{O}_{12}{ }^{9}$, lead magnesium niobate-lead titanate, ${ }^{10}$ lead-zirconate titanate ${ }^{11}$ or conductive particles such as expanded graphite, ${ }^{12}$ PANI encapsulated particles in polydivinylbenzene, ${ }^{13,14}$ carbon nanotubes, ${ }^{15}$ polythiophene, ${ }^{16}$ and $\mathrm{Ag}$ nanoparticles ${ }^{17}$ have been used. In most of the cases, an increase in the permittivity was accompanied by an increase in the dielectric loss, which resulted in a decrease of the dielectric breakdown field. Furthermore, because the increase in permittivity occurred at fillers concentrations approaching the percolation threshold, the mechanical properties of the composites were also affected.

A second strategy used is to chemically modify the silicones with polar groups. ${ }^{18}$ These polar groups were either introduced as side groups or as cross-linkers. ${ }^{5}$ In many cases, due to the difficulty in cross-linking such polar silicones to elastic materials with good properties, the modified silicones were rather used as high permittivity fillers in silicone matrices with good mechanical properties. ${ }^{19}$ Due to the strong polarity difference of the two components of the blend, the high permittivity polar silicone filler and the non-polar polydimethylsiloxane matrix phase separate and nonhomogenous materials form. As for now, the permittivity of such blended materials was increased up to 7. For some applications, however, this value is still too low. When the polar groups attached to the cross-linker, homogenous materials form, but the amount of polar groups that can be introduced is limited and thus the increase in the permittivity is even lower than $7 .^{20}$

Since the permittivity of an elastomer is usually increased with a higher dipole concentration, it would be advantageous to increase the concentration of polar groups in silicone as much as possible. This can be achieved by replacing one methyl group of the dimethylsiloxy unit with a polar side group. This approach allows the formation of polysiloxanes that carries a polar group at every repeat unit. Work in this direction was recently published concerning silicones modified with $\mathrm{CN}^{21,22}$ and with $\mathrm{Cl}^{23}{ }^{22}$

In this preprint, a variety of new silicone materials modified with polar $\mathrm{CN}$ and $\mathrm{CF}_{3}$ groups are presented. The advantages and disadvantages of these materials are discussed.

\section{RESULTS AND DISCUSSION}

The permittivity of an elastomer can be increased by a modification with polar groups. We focused on $\mathrm{CN}$ and on $\mathrm{CF}_{3}$ groups because they have a large dipole moment and a low volume which will allow them to be easily polarized in an electric field over a wide range of frequencies. Polysiloxanes containing CN groups were synthesized via anionic polymerization of nitrile containing cyclosiloxanes, via a post-polymerization modification of functional polysiloxanes containing either hydrosilyl or vinyl groups, and via a nitrile functionalized cross-linker. Polysiloxanes containing $\mathrm{CF}_{3}$ groups were prepared by anionic co-polymerization of 1,3,5-tris(3,3,3-trifluoropropyl)-1,3,5-trimethylcyclosiloxane $\left(\mathrm{F}_{3}\right)$ with octamethylcyclotetrasiloxane $\left(\mathrm{D}_{4}\right)$.

We will first describe the synthesis of silicone elastomers containing $\mathrm{CN}$ groups, then the silicone containing $\mathrm{CF}_{3}$ groups, and finally, we will compare the most promising new materials with the well-known VHB foil and with Elastosil silicone.

\subsection{Anionic polymerization of nitrile containing cyclosiloxanes}

Nitrile containing silicones can in principle be prepared by ring opening polymerization of cyclosiloxanes that carry $\mathrm{CN}$ groups either in cationic or anionic conditions. Kinetically controlled polymerization can be achieved with monomers that have ring strain. Such monomers are difficult to synthesize but they allow formation of polymers in high yields. ${ }^{24}$ The polymerization of cyclosiloxanes with no ring strain is thermodynamically controlled. At equilibrium a mixture of polymers and small cycles is formed.

Two silicone monomers modified with $\mathrm{CN}$ groups were prepared (Scheme 1). Photo-induced thiol-ene addition of 3mercaptopropionitrile to 2,4,6,8-tetramethyl-2,4,6,8-tetravinylcyclotetrasiloxane $\left(\mathrm{V}_{4}\right)(\mathbf{1})$ allows formation of monomer 2 in one step in $92 \%$ yield. By using a slight excess of thiol, full conversion of the vinyl groups was achieved under few min of UV-irradiation. Hydrosilylation reaction of allyl cyanide to 2,4,6,8-tetramethylcyclosiloxane $\left(\mathrm{D}_{4} \mathrm{H}\right)$ (4) in 
presence of a platinum catalyst allows the formation of monomer 5. To achieve full conversion of the hydrosilane groups, the reaction was refluxed in toluene for 5 days with excess allyl cyanide. The high reaction temperature and the long reaction time led to some degradation of the product. Monomer 5 was prepared in $92 \%$ yield.
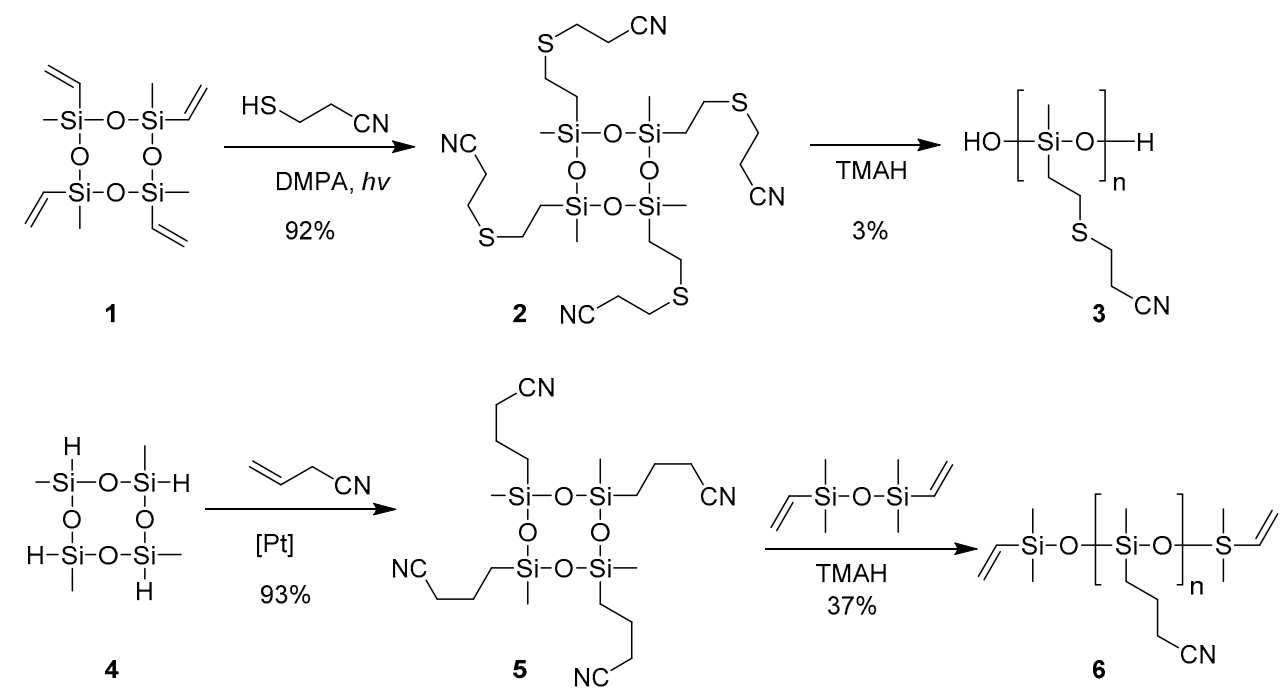

Scheme 1: Synthesis of nitrile containing cyclosiloxanes and their polymerization.

For both reactions, most of the addition product was anti-Markovnikov, but a small amount of Markovnikow addition product was also observed. Based on ${ }^{1} \mathrm{H}$ NMR analysis, the thiol-ene addition gives about $6 \%$ Markovnikow adduct, which is in agreement with literature, ${ }^{25}$ while the hydrosilylation reaction is less selective and gives about $20 \%$ of this adduct. It was not possible to separate these regioisomers by column chromatography and therefore mixtures were used for polymerization.

Anionic ring opening polymerizations of $\mathbf{2}$ and $\mathbf{5}$ in presence of tetramethylammonium hydroxide (TMAH) gave the respective polymers in rather low yields. The content of polymer and small cycles was determined by GPC (Figure 1). Monomer 2 gave about 3\% polymer $\left(M_{w}=35 \mathrm{kDa}\right)$, while monomer 5 with sterically less demanding side groups gave a polymer yield of $37 \%\left(M_{w}=17 \mathrm{kDa}\right)$. It is known that the side groups of cyclosiloxanes can influence significantly the equilibrium between the linear polymer and the low molecular weight cycles. A trend towards increased amount of cycles was found for sterically demanding and electron withdrawing groups by Wright and Semlyen. ${ }^{26}$ Due to the low polymer yield the synthesis of polysiloxanes from cyclosiloxanes modified with polar nitrile groups appeared to be unattractive.
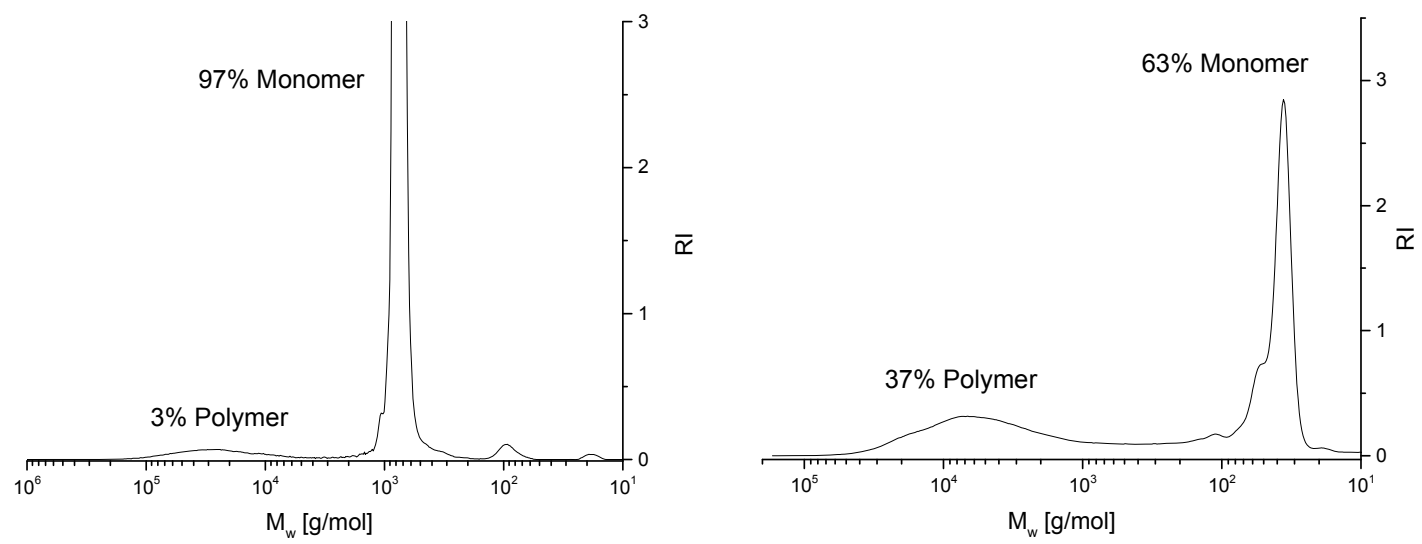

Figure 1: GPC chromatograms of the reaction mixture obtained from polymerization of 2 (left) and 4 (right) in presence of TMAH. 


\subsection{Post-polymerization modification of functional polysiloxanes containing either vinyl or hydrosilyl groups}

Polysiloxanes containing $\mathrm{CN}$ groups were synthesized via post-polymerization modifications of functional polysiloxanes containing either hydrosilyl P1 or vinyl groups P2. The synthesis started either from P1 and a mixture of allyl cyanide/hexene or from P2 and a mixture of 3-mercaptopropionitrile/butanethiol, to afford polymers P1(x:y) and P2(x:y) with different content of polar CN- (x) and butylthioether (y) groups, respectively (Scheme 2). Hydrosilylation unfortunately did not allow the quantitative conversion of all hydrosilyl groups. ${ }^{27}$ It also required elevated temperatures, long reaction times, and expensive Pt catalyst. The unreacted hydrosilane groups participated in uncontrolled side reactions with formation of a cross-linked material. In contrast, the latter reaction is fast, requires no expensive catalyst, and proceeds to completion when a slight excess of thiol to vinyl groups is used. ${ }^{18}$ The only disadvantage is the pungent odor of the thiols and their instability.

The concentration of polar $\mathrm{CN}$ groups was fine-tuned and the resulting changes in the glass transition temperature $\left(T_{g}\right)$ and the dielectric permittivity were monitored. An almost linear increase in $T_{g}$ and in permittivity was observed with enhanced CN content (Figure 2). Fortunately, all prepared polymers have a $T_{g}$ well below room temperature which should in principle allow their cross-linking to elastic materials. The maximum permittivity $\varepsilon^{\prime}=18.2$ as well as the highest $T_{g}=-52{ }^{\circ} \mathrm{C}$ was observed for the polysiloxane modified at every repeat unit with a polar $\mathrm{CN}$ group.

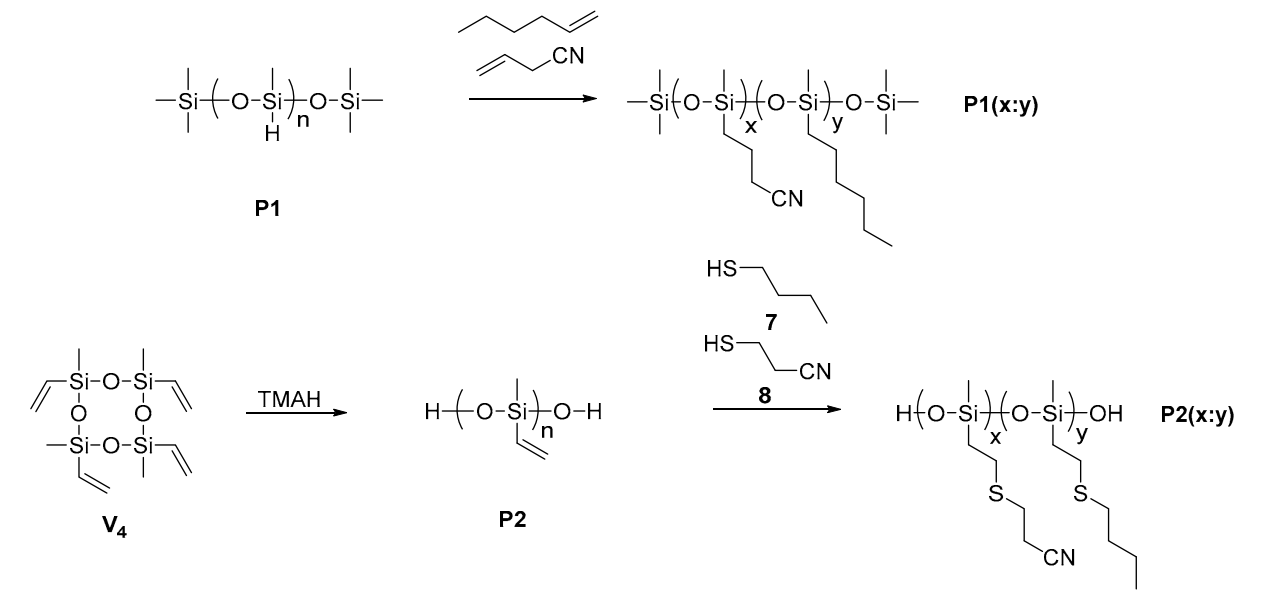

Scheme 2: Synthesis of polysiloxanes modified with polar CN groups via hydrosilylation (top) and thiol-ene additions (bottom).

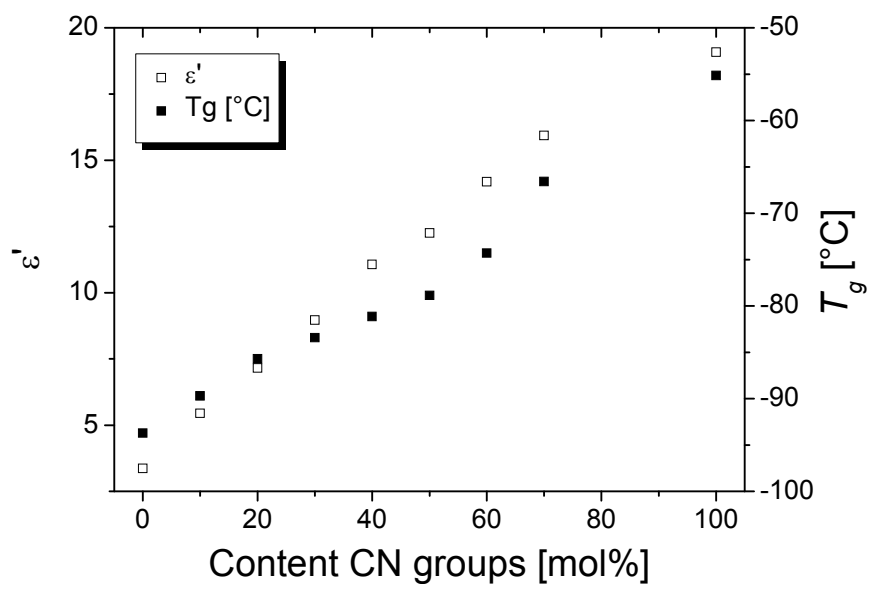

Figure 2: Dielectric permittivity at $10 \mathrm{kHz}$ and $T_{g}$ of polysiloxanes P2(x:y) prepared via thiol-ene reaction as function of $\mathrm{mol} \%$ of polar $\mathrm{CN}$ groups. 


\subsection{Silicone based elastomers modified with polar $\mathrm{CN}$ groups}

Three different approaches were followed to prepare silicone elastomers containing polar $\mathrm{CN}$ groups. The first one uses a functional cross-linker that carries a $\mathrm{CN}$ group, ${ }^{28}$ the second one uses a silicone modified with polar $\mathrm{CN}$ groups as high permittivity filler and cross-linker, ${ }^{29,30}$ while the third approach uses a one step process in which the polar CN groups and the cross-linking occur simultaneously. ${ }^{21}$

In the first approch, a hydroxyl end-functionalized polydimethylsiloxane $\left(M_{\mathrm{W}}=139 \mathrm{kDa}\right)$ was cross-linked via a condensation reaction with varying amounts of 3-cyanopropyltriethoxysilane (Scheme 3). The functionalized crosslinker can participate in a condensation reaction with the hydroxyl end-groups of the silicone matrix but can also undergo self-condensation reactions under formation of silsesquioxane structures. The in situ generated silsesquioxanes enable varying the amount of $\mathrm{CN}$ groups in the material from $25 \mathrm{wt} \%$ to $50 \mathrm{wt} \%$. The permittivity and mechanical properties of the formed elastomers are given in Figure 3.

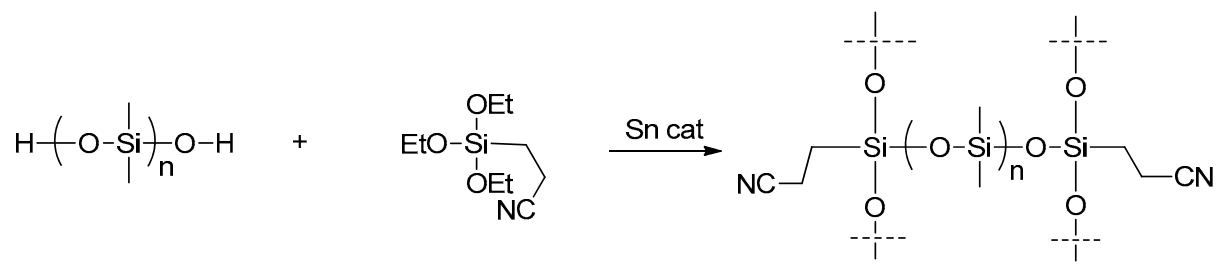

Scheme 3: Synthesis of silicone elastomers starting from a hydroxyl end-functionalized polydimethysiloxane and 3cyanopropyltriethoxysilane. Silsesquioxanes are generated in situ (not shown).
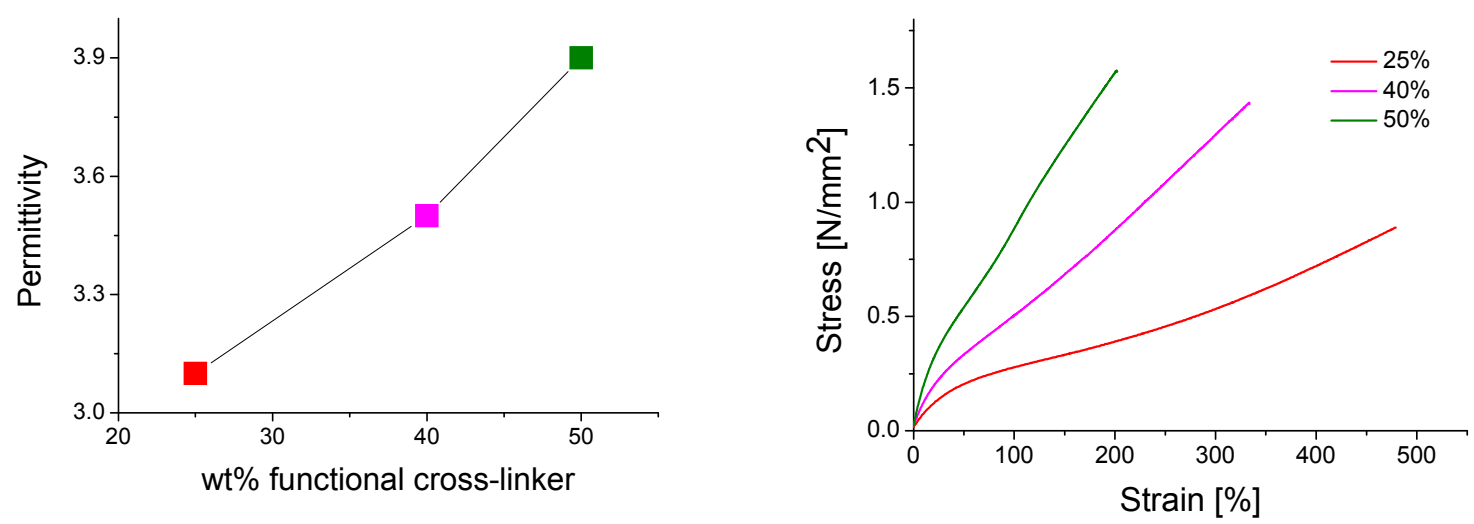

Figure 3: Dielectric permittivity at $30 \mathrm{kHz}$ (left) and stress-strain curves (right) of elastomers prepared by cross-linking a hydroxyl end-functionalized polydimethylsiloxane with different $\mathrm{wt} \%$ of 3 -cyanopropyltriethoxysilane.

Surprisingly, despite the large amount of cross-linker used, the increase in permittivity was only moderate $\left(\varepsilon_{\max }^{\prime}=3.9\right)$. The reason behind this might be the formation of silsesquioxane structures which entrap the $\mathrm{CN}$ groups hence hindering their polarization in an electric field. As expected, the materials are becoming stiffer and the strain at break is reduced with increasing cross-linker content. Investigations regarding the electromechanical properties of these materials are on the way.

In the second approach, polysiloxanes P1(x:y) were used as high permittivity fillers. These fillers were either crosslinked into interpenetrating networks with a polydimethylsiloxane matrix or processed into nanoparticles that were blended into a polydimethylsiloxane matrix with good mechanical properties. ${ }^{30}$ This approach led to novel materials with excellent mechanical properties and high breakdown fields $\left(E_{b}=56 \mathrm{~V} / \mu \mathrm{m}\right)$. The level to which the permittivity increased $\left(\varepsilon^{\prime}=4.5\right)$ leaves space for further improvement.

In the last approach, the modification of polysiloxane with $\mathrm{CN}$ groups and the cross-linking were carried out simultaneously in a one step process (Scheme 4). ${ }^{21}$ This allowed us the preparation of elastic materials with remarkable 
dielectric, mechanical, and electromechanical properties which turned out to be extremely robust and fully reproducible. Additionally, it allowed us to adjust the dielectric properties of the formed elastomers $\mathbf{E}_{\mathbf{C N}}(\mathbf{x}: \mathbf{y})$, where x:y represents the ratio of $\mathrm{CN}$ : butylthioether groups, just by using different ratios of 3-mercaptopropionitrile/butanethiol. This chemical modification has a direct impact on the actuation strain.

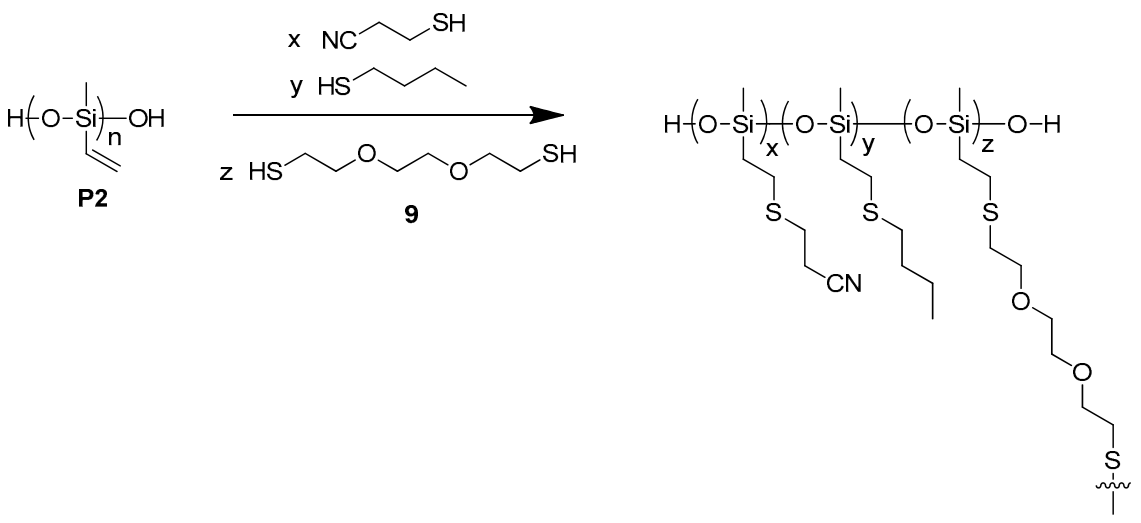

Scheme 4: One step process to silicone elastomers $\mathbf{E}_{\mathbf{C N}}(\mathbf{x}: \mathbf{y})$ having different mol\% of polar $\mathrm{CN}$ groups.

When a solution of a polymethylvinylsiloxane P2, mercaptopropionitrile 7, cross-linker 9, radical initiator 2,2dimethoxy-2-phenylacetophenone (DMPA), and 5\% of hexamethyl disilazane treated $\mathrm{SiO}_{2}$ nanoparticles in THF was casted on a Teflon substrate and irradiated with a UV-lamp, a thin film formed. IR investigations show that the surface exposed to air contains some unreacted vinyl groups (Figure 4). ${ }^{21}$ To avoid radical quenching by oxygen, further optimizations were conducted for the processing of materials $\mathbf{E}_{\mathbf{C N}}(\mathbf{x}: \mathbf{y})$ into thin films. The top surface of a casted precursor solution on Teflon was covered by a glass plate, whereby precision spacers were used to control the thickness of the film (Figure 4). The IR spectra of P2 as well as of P2(1:0) are also included in Figure 4. The conversion of the vinyl groups in the film was investigated by FT-IR measurements conducted on the glass and Teflon exposed surfaces as well as on pellets obtained by pressing the powders of ground films in $\mathrm{KBr}$. The vinyl group has three characteristic signals at $3055 \mathrm{~cm}^{-1}, 3020 \mathrm{~cm}^{-1}$ and $2965 \mathrm{~cm}^{-1}$ with the one at $3055 \mathrm{~cm}^{-1}$ having the highest intensity. The signal at 2925 $\mathrm{cm}^{-1}$ is characteristic for the nitrile groups. By using a top glass plate in thin film formation, the oxygen quenching was significantly reduced and an increase in the conversion of the vinyl groups was observed.

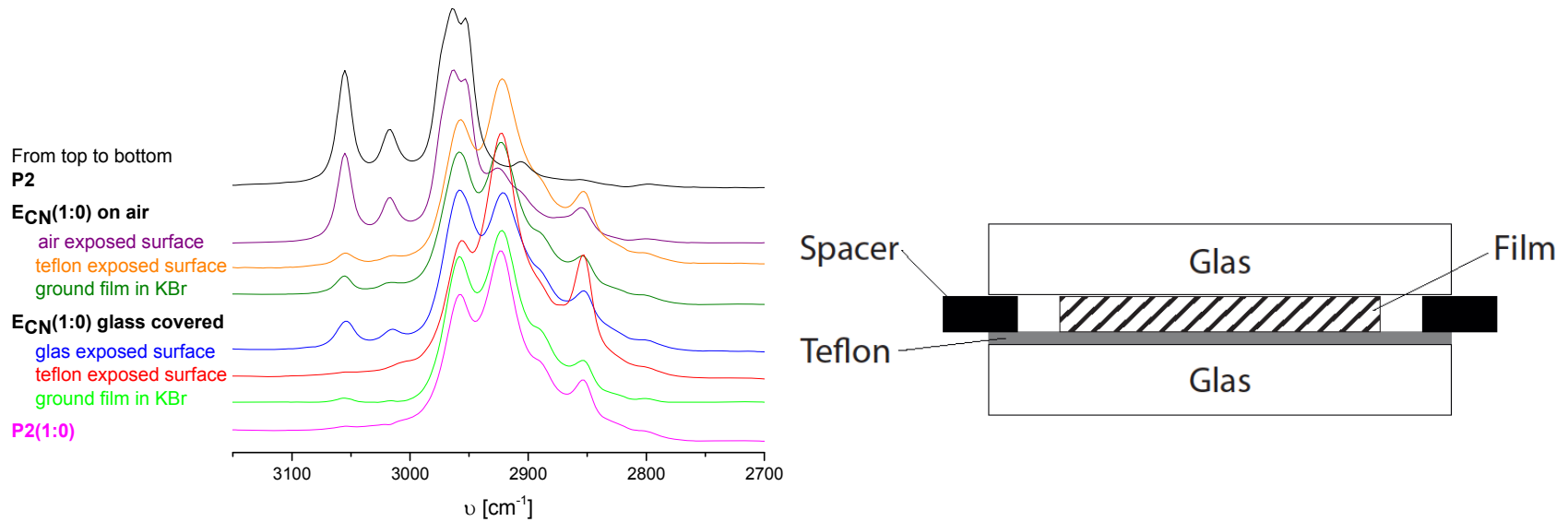

Figure 4: IR spectra of $\mathbf{P 2}, \mathbf{E}_{\mathbf{C N}}\left(\mathbf{1 : 0 )}\right.$ prepared on Teflon substrate, $\mathbf{E}_{\mathbf{C N}}(\mathbf{1 : 0 )}$ prepared between a Teflon and a glass plate, and uncross-linked P2(1:0) (left) as well as the setup used for the formation of $\mathbf{E}_{\mathbf{C N}}(\mathbf{x}: \mathbf{y})$ (right).

The new setup further improved the processability of the elastomer films and has a direct influence on the dielectric properties of the formed materials. For example, for the case where we aimed at elastomers with maximum content of CN groups $\mathbf{E}_{\mathbf{C N}}(\mathbf{1 : 0})$, a film cured in air showed a permittivity value of 10.1 at $10 \mathrm{kHz}$ and a relaxation peak at $500 \mathrm{~Hz}$ 
that results in a further increase in permittivity, ${ }^{21}$ while the film cured with the optimized method allowed formation of a material with permittivity as high as $\varepsilon^{\prime}=17.7$ at $10 \mathrm{kHz} .^{31}$

The permittivity of $\mathbf{E}_{\mathbf{C N}}(\mathbf{x}: \mathbf{y})$ can be fine-tuned by using a mixture of mercaptopropionitrile/butanethiol. For example, the dielectric permittivity at high frequencies increased from $\varepsilon^{\prime}=4.3$ for $\mathbf{E}_{\mathbf{C N}}(\mathbf{0 : 1})$, to $\varepsilon^{\prime}=9.7$ for $\mathbf{E}_{\mathbf{C N}}(\mathbf{1 : 1})$, to a maximum value of $\varepsilon^{\prime}=17.5$ for $\mathbf{E}_{\mathbf{C N}}(\mathbf{1 : 0})$ which has a $\mathrm{CN}$ group at every repeat unit (Figure 5). Materials $\mathbf{E}_{\mathbf{C N}}(\mathbf{x}: \mathbf{y})$ were investigated in electromechanical tests. A linear increase in the actuation strain at $10 \mathrm{~V} / \mu \mathrm{m}$ with increasing the content of CN groups was observed from $2.6 \%$ for $\mathbf{E}_{\mathbf{C N}}(\mathbf{0 : 1})$, to $6.4 \%$ for $\mathbf{E}_{\mathbf{C N}}\left(\mathbf{1 : 1 )}\right.$, to a value of $14.2 \%$ for $\mathbf{E}_{\mathbf{C N}}(\mathbf{1 : 0})$ (Figure 5). Additionally, some of the actuators can self-repair after a breakdown which increases device lifetime and reliability. Given the favourable conditions of these materials, we now consider it a not too far-fetched option to construct devices for implantation into humans.
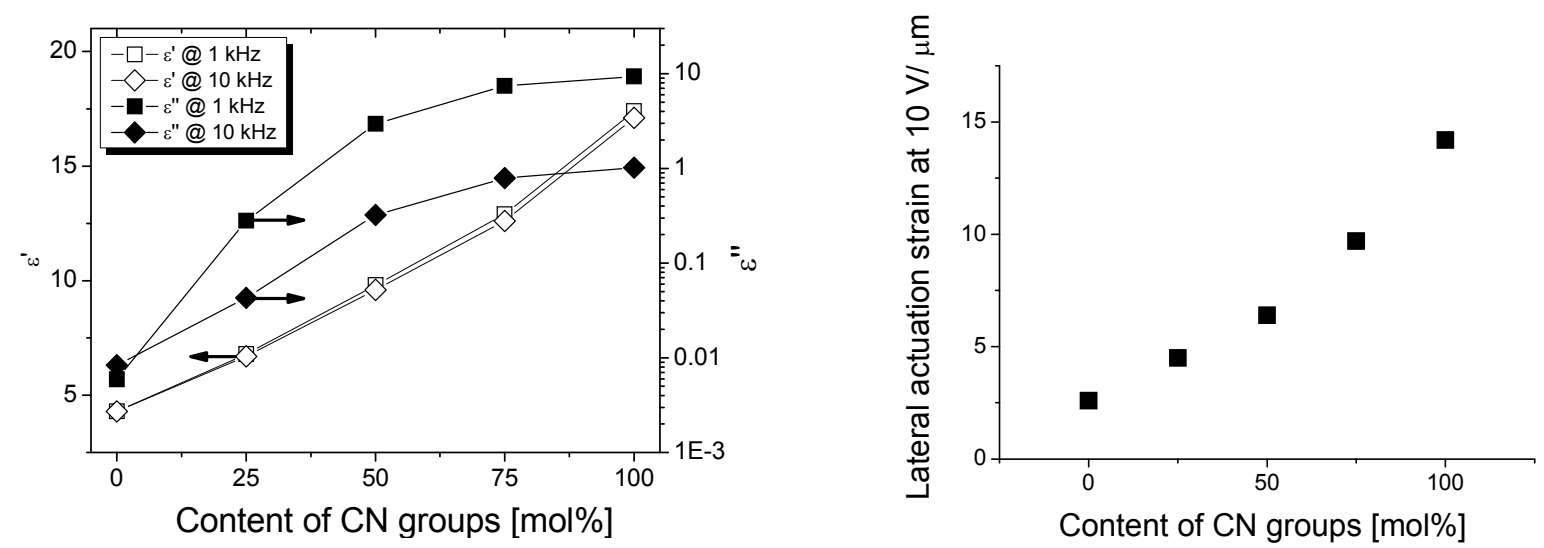

Figure 5: Dielectric permittivity and dielectric loss at $1 \mathrm{kHz}$ and at $10 \mathrm{kHz}$ (left) and the influence the content of $\mathrm{CN}$ groups has on the lateral actuation strain at $10 \mathrm{~V} / \mu \mathrm{m}$ (right) for materials $\mathbf{E}_{\mathbf{C N}}(\mathbf{x}: \mathbf{y})$.

\subsection{Silicone based elastomers modified with polar $\mathrm{CF}_{3}$ groups}

Polysiloxane elastomers containing varying $\mathrm{mol} \%$ of $\mathrm{CF}_{3}$ groups, $\mathbf{E}_{\mathbf{C F} 3}(\mathbf{x})$, were prepared via anionic ring opening polymerization of $\mathrm{F}_{3}$ and $\mathrm{D}_{4}$ in presence of 1,3-divinyl-1,1,3,3-tetramethyldisiloxane end-blocker (Scheme 5). ${ }^{32}$ Vinyl end-groups were introduced to the polymers which allowed us to cross-link them into thin films. The amount of $\mathrm{CF}_{3}$ groups in the materials was varied from 28 to $58 \mathrm{~mol} \%$. By increasing the content of $\mathrm{CF}_{3}$ groups, an increase in permittivity from $\varepsilon^{\prime}=5.1$ for $\mathbf{E}_{\mathbf{C F 3} 3}(\mathbf{2 8})$ to $\varepsilon^{\prime}=6.4$ for $\mathbf{E}_{\mathbf{C F} 3}(\mathbf{5 8})$, as well as an increase in the $T_{g}$ from $-106{ }^{\circ} \mathrm{C}$ for $\mathbf{E}_{\mathbf{C F} 3}(\mathbf{2 8})$ to $-84.2{ }^{\circ} \mathrm{C}$ for $\mathbf{E}_{\mathbf{C F 3} 3}(\mathbf{5 8})$ was observed (Figure 6).

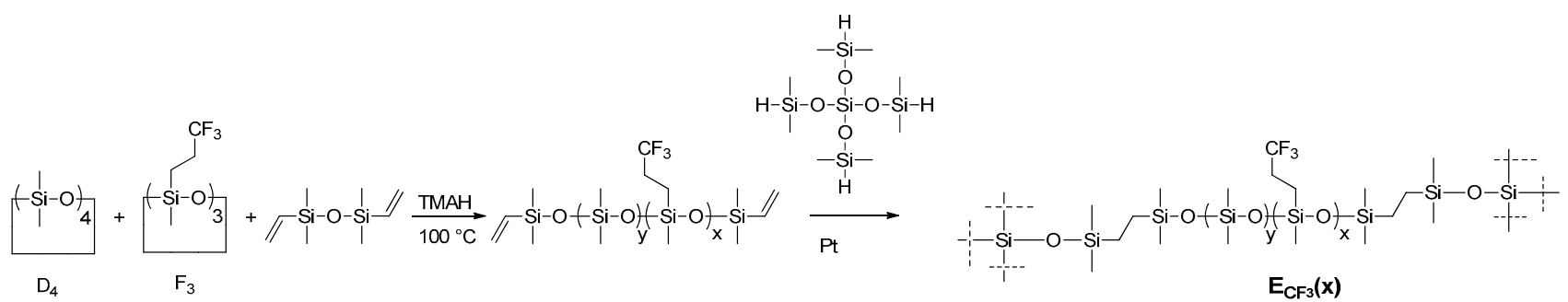

Scheme 5. Synthesis of polysiloxanes containing $\mathrm{CF}_{3}$ groups starting from $\mathrm{D}_{4}, \mathrm{~F}_{3}$, and 1,3-divinyl-1,1,3,3tetramethyldisiloxane and their cross-linking to elastic materials $\mathbf{E}_{\mathbf{C F 3}}(\mathbf{x})$ via a hydrosilylation reaction of the vinyl groups with tetrakis(dimethysiloxy)silane. 


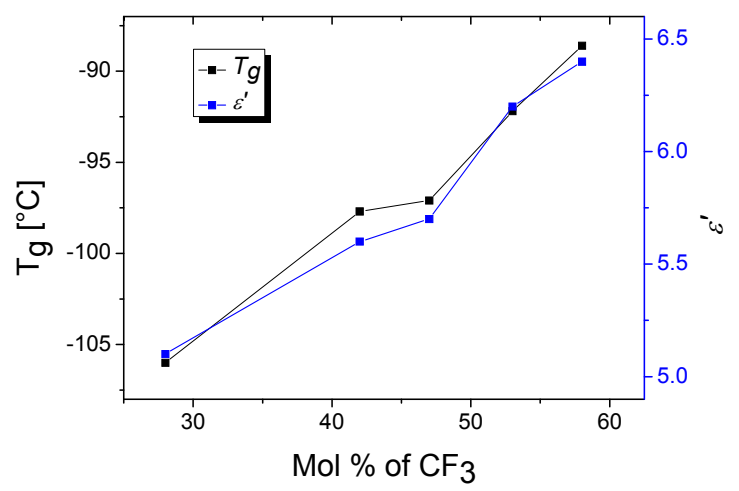

Figure 6: Glass transition temperature $\left(T_{g}\right)$ and dielectric permittivity of $\mathbf{E}_{\mathbf{C F 3}}(\mathbf{x})$ as function of mol\% of $\mathrm{CF}_{3}$ groups.

Unfortunately, the $M_{w}$ of the polymers containing different amount of $\mathrm{CF}_{3}$ varied from $49 \mathrm{kDa}$ to $140 \mathrm{kDa}$. Therefore a direct comparison of the influence the content of $\mathrm{CF}_{3}$ groups in different materials has on the mechanical properties was not possible because more than one parameter was changed. However, it was noted that $\mathrm{CF}_{3}$ groups have a plasticising effect. For example material $\mathbf{E}_{\mathbf{C F} 3}(\mathbf{5 3})$ is much softer as compared to $\mathbf{E}_{\mathbf{C F} 3}(\mathbf{2 8})$ despite that the $M_{w}$ of $\mathbf{E}_{\mathbf{C F} 3}(\mathbf{5 3})$ was lower as compared to $\mathbf{E}_{\mathrm{CF}_{3}}(\mathbf{2 8})$. A strong reduction of the electric field required to induce the electromechanical work was observed for material $\mathbf{E}_{\mathbf{C F} 3} \mathbf{( 5 3 )}$ mainly due to the increased $\varepsilon^{\prime} / Y$ value. (Figure 7)
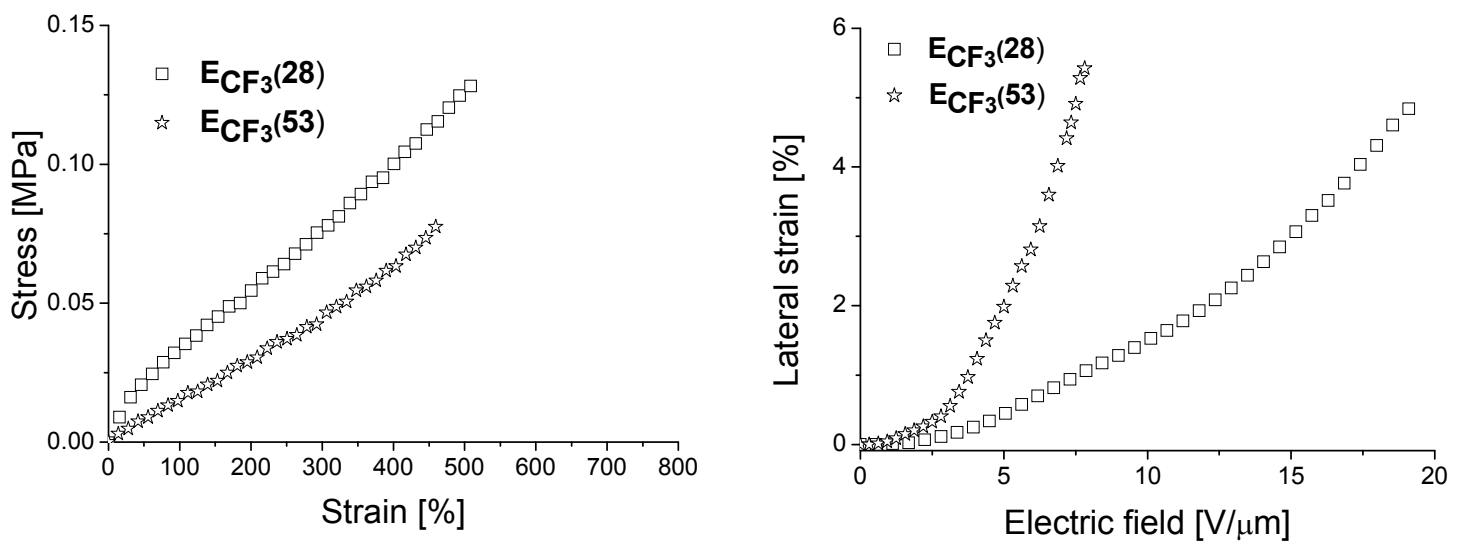

Figure 7: Stress-strain curves (left) and lateral actuation strain as function of electric field of materials $\mathbf{E}_{\mathbf{C F 3}}(\mathbf{x})$ (right).

\subsection{Empa materials versus regular silicone and VHB}

The properties of some materials prepared in our group at Empa, $\mathbf{E}_{\mathbf{C N}}\left(\mathbf{1 : 0 )}\right.$ and $\mathbf{E}_{\mathbf{C F} 3}(\mathbf{5 3})$, were compared with two common elastomer materials: a commercial polydimethylsiloxane (PDMS, Elastosil RT 746) and the acrylic foil VHB 4905 from 3M. The dielectric properties as function of frequency are shown in Figure 8. The permittivity of different materials at high frequencies was $\varepsilon^{\prime}=3$ for PDMS, $\varepsilon^{\prime}=4.4$ for $\mathrm{VHB}, \varepsilon^{\prime}=6.2$ for $\mathbf{E}_{\mathbf{C F} 3}(\mathbf{5 3})$, and $\varepsilon^{\prime}=17.5$ for $\mathbf{E}_{\mathbf{C N}}(\mathbf{1 : 0})$. The lowest conductivity at low frequencies was measured for VHB and for PDMS, and an increase in the conductivity was observed for $\mathbf{E}_{\mathbf{C N}}\left(\mathbf{1 : 0 )}\right.$ and $\mathbf{E}_{\mathbf{C F 3}}(\mathbf{5 3})$ (Table 1). 


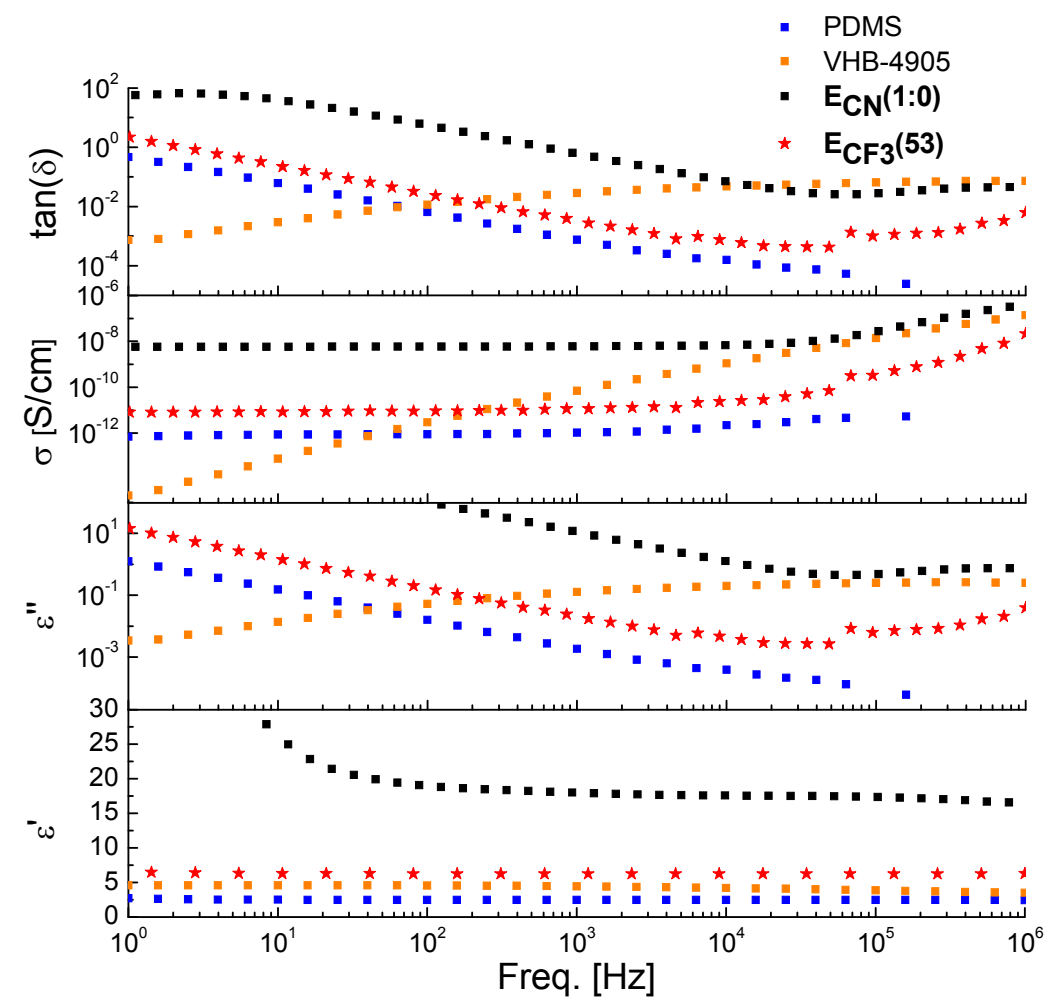

Figure 8: Dielectric permittivity $\varepsilon$ ', dielectric loss $\varepsilon "$, conductivity $\sigma$, and loss factor $\tan (\delta)$ as function of frequency of selected materials $\mathbf{E}_{\mathbf{C N}}\left(\mathbf{1 : 0 )}\right.$ and $\mathbf{E}_{\mathbf{C F 3}} \mathbf{( 5 3 )}$ as well as of commercial silicone Elastosil RT 746 (PDMS) and VHB-4905.

The stress-strain curves of PDMS, VHB, and the two selected materials are presented in Figure 9. By far the highest strain at break is observed for Elastosil (770\%) and VHB (850\%), while material $\mathbf{E}_{\mathbf{C F} 3}(\mathbf{5 3})$ and $\mathbf{E}_{\mathbf{C N}} \mathbf{1 : 0 )}$ showed a lower strain at break of $460 \%$ and $170 \%$, respectively. Compared to the commercial PDMS and VHB 4905, the polar silicones $\mathbf{E}_{\mathbf{C F 3}}(\mathbf{5 3})$ is softer while material $\mathbf{E}_{\mathbf{C N}}(\mathbf{1 : 0 )}$ is significantly stiffer.
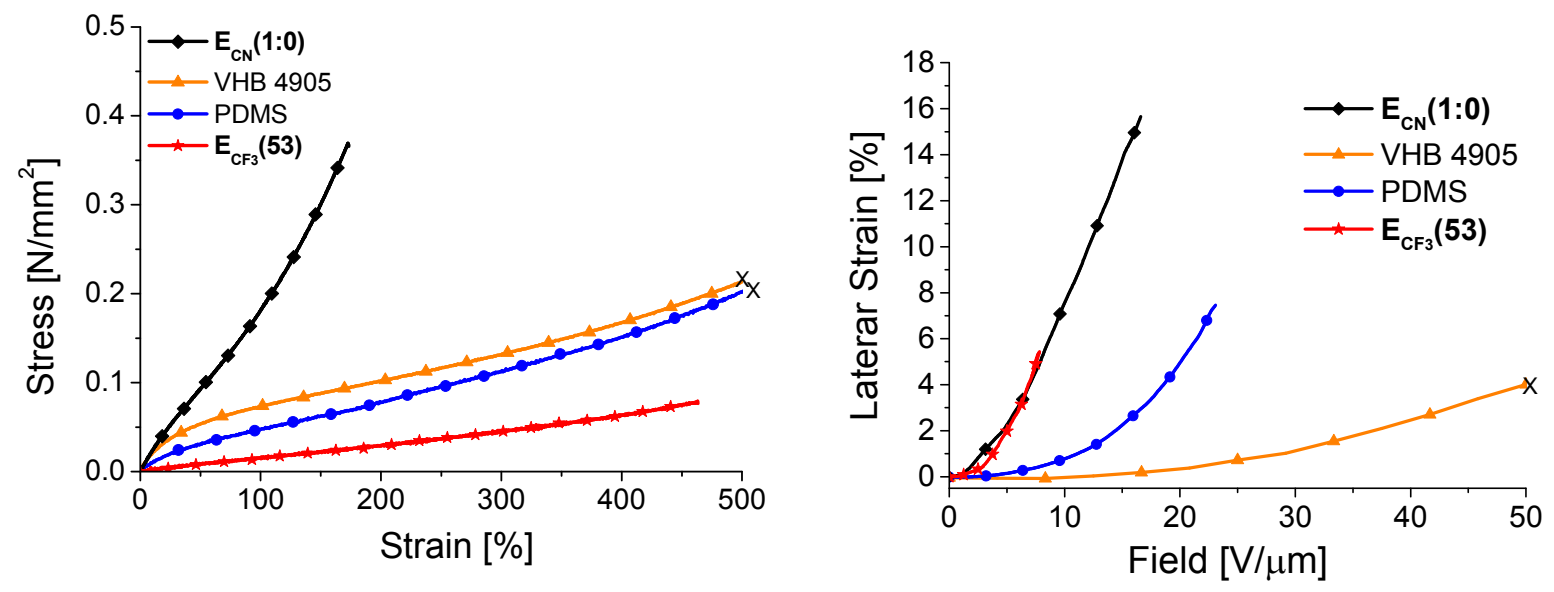

Figure 9: Stress-strain curves (left) and lateral actuation strain as function of electric field (right) for selected materials synthesized in our lab and for commercial silicone Elastosil RT 746 (PDMS) and VHB-4905. 
The results obtained from the circular membrane actuators are presented in Figure 9 right. A stepwise increasing voltage was applied on the actuator and the lateral actuation was measured. Table 1 summarizes the elastic moduli at $10 \%$ and $50 \%$ strain, the permittivity at $10 \mathrm{kHz}, \varepsilon^{\prime} / Y_{10 \%}$ ratio, actuator breakdown field $\left(E_{b}\right)$, lateral actuation strain at $7.8 \mathrm{~V} / \mu \mathrm{m}$, and the electric field required for different dielectric materials to produce $7 \%$ lateral strain. Materials $\mathbf{E}_{\mathbf{C F} 3}(\mathbf{5 3})$ and $\mathbf{E}_{\mathbf{C N}}(\mathbf{1 : 0})$ showed by far the highest lateral actuation strain at the lowest electric field of $5.4 \%$ at only $7.8 \mathrm{~V} / \mu \mathrm{m}$, while PDMS gave only $0.5 \%$ strain and VHB does not actuate at all at such low voltages. While material's $\mathbf{E}_{\mathbf{C F} 3}(\mathbf{5 3})$ improved actuation strain is due to the increase in permittivity and the low elastic modulus $\left(\varepsilon^{\prime} / Y_{10 \%}=310 \mathrm{MPa}^{-1}\right)$, material's $\mathbf{E}_{\mathbf{C N}}(\mathbf{1 : 0 )}$ improved actuation strain is only due to the high dielectric permittivity, since this material was the stiffest of the four.

Table 1. Elastic moduli at different strain levels, the dielectric permittivity $\varepsilon^{\prime}$, the $\varepsilon^{\prime} / Y_{10 \%}$ ratio, conductivity $\sigma$, actuator breakdown field $E_{b}$, lateral actuation strain at $7.8 \mathrm{~V} / \mu \mathrm{m}$, and the electric field required to induce $7 \%$ lateral actuation strain.

\begin{tabular}{lcccccccc}
\hline Entry & $\begin{array}{c}Y_{10 \%} \\
{[\mathrm{kPa}]}\end{array}$ & $\begin{array}{c}Y_{50 \%} \\
{[\mathrm{kPa}]}\end{array}$ & $\varepsilon^{\prime}$ & $\begin{array}{c}\varepsilon^{\prime} / Y_{10 \%} \\
{\left[\mathrm{MPa}^{-1}\right]}\end{array}$ & $\begin{array}{c}\sigma \\
{[\mathrm{S} / \mathrm{cm}]}\end{array}$ & $\begin{array}{c}E_{b} \\
{[\mathrm{~V} / \mu \mathrm{m}]}\end{array}$ & $\begin{array}{c}{ }^{\mathrm{a}} \text { Actuation } \\
{[\%]}\end{array}$ & $\begin{array}{c}{ }^{\mathrm{b}} E \\
{[\mathrm{~V} / \mu \mathrm{m}]}\end{array}$ \\
\hline $\mathbf{E}_{\mathbf{C N}} \mathbf{( 1 : 0 )}$ & 155 & 96.2 & 17.5 & 112.9 & $5.77 \times 10^{-9}$ & 11.8 & $5.4 \%$ & $9.65 \mathrm{~V} / \mu \mathrm{m}$ \\
$\mathbf{E}_{\mathbf{C F 3}}(\mathbf{5 3})$ & 20 & 17 & 6.2 & 310.0 & $2.03 \times 10^{-11}$ & 7.8 & $5.4 \%$ & - \\
Elastosil & 79 & 41 & 3 & 38.0 & $1.75 \times 10^{-16}$ & 25 & $0.5 \%$ & $22.5 \mathrm{~V} / \mu \mathrm{m}$ \\
VHB & 150 & 50 & 4.4 & 29.3 & $2.95 \times 10^{-15}$ & $>100$ & $0 \%$ & $61.3 \mathrm{~V} / \mu \mathrm{m}$ \\
\hline
\end{tabular}

${ }^{a}$ Lateral actuation strain at $7.8 \mathrm{~V} / \mu \mathrm{m}$

${ }^{\mathrm{b}}$ Electric field required to produce $7 \%$ lateral strain.

\section{CONCLUSION}

We have shown that the permittivity of silicone elastomers can be fine-tuned by modification with polar $\mathrm{CN}$ and $\mathrm{CF}_{3}$ groups. One of the $\mathrm{CN}$-modified materials obtained shows a powerful combination of properties: $\varepsilon^{\prime}=17.5, Y_{10 \%}=155$ $\mathrm{kPa}$, and strain at break of $170 \%$ while a material that contains $53 \mathrm{~mol} \% \mathrm{CF}_{3}$ groups shows a $\varepsilon^{\prime}=6.2, Y_{10 \%}=20 \mathrm{kPa}$, and strain at break of $460 \%$. The actuation strain at low electric fields was significantly enhanced for these modified silicones. Both materials show an actuation strain of $5.4 \%$ at an electric field of $7.8 \mathrm{~V} / \mu \mathrm{m}$. At this electric field the material modified with $\mathrm{CF}_{3}$ groups suffers dielectric breakdown, while the material modified with $\mathrm{CN}$ groups withstands higher electric fields of $16.5 \mathrm{~V} / \mu \mathrm{m}$. At this field, a maximum lateral actuation strain of $15.6 \%$ was measured. The high actuation strain at the low electric field is mainly due to the high permittivity $\varepsilon^{\prime}=17.5$ of this material. Additionally, some of the actuators can self-repair after a breakdown which increases device lifetime and reliability. The materials modified with $\mathrm{CF}_{3}$ groups show significantly higher strain at break as compared with the materials containing $\mathrm{CN}$ groups. While an increase in stiffness with higher $\mathrm{CN}$ content in elastomers was observed, the $\mathrm{CF}_{3}$ materials turn softer with higher $\mathrm{CF}_{3}$ content. These latter materials, however, show only a moderate increase in permittivity.

Based on these findings we expect that a material formed by cross-linking a copolymer with both $\mathrm{CN}$ and $\mathrm{CF}_{3}$ groups will have properties that lie in between the properties of both homopolymers. Future work is going to explore this. Given the simplicity of the synthetic protocols used for the synthesis of these silicone elastomers, we expect these materials to be scalable and to find their way into applications. Because of the low actuation voltage achieved, we now consider these actuators a not too far-fetched option to construct devices for implantation into humans.

\section{ACKNOWLEDGEMENTS}

We gratefully acknowledge Swiss National Science Foundation (SNF132101, Swiss-Romanian Cooperation Program, grant No. IZERZO_142215 / 1), Sciex (12.192), and Swiss Federal Laboratories for Materials Science and Technology (Empa, Dübendorf) for financial support. 


\section{REFERENCES}

[1] Pelrine, R., Kornbluh, R., Kofod, G., "High-strain actuator materials based on dielectric elastomers," Adv. Mater. 12(16), 1223-1225 (2000).

[2] Pelrine, R., Kornbluh, R., Pei, Q., Joseph, J., "High-Speed Electrically Actuated Elastomers with Strain Greater Than 100\%," Science. 287(5454), 836-839 (2000).

[3] Carpi, F., [Introduction to transducers and artificial muscles based on Electromechanically Active Polymers], in press.

[4] Romasanta, L. J., Lopez-Manchado, M. A., Verdejo, R., "Increasing the performance of dielectric elastomer actuators: A review from the materials perspective," Prog. Polym. Sci. 51, 188-211 (2015).

[5] Madsen, F. B., Daugaard, A. E., Hvilsted, S., Skov, A. L., "The Current State of Silicone-Based Dielectric Elastomer Transducers," Macromol. Rapid Commun. preprint, DOI: 101002/marc.201500576 (2016).

[6] Opris, D. M., Quinsaat, J. E. Q., Dünki, S., Ko, Y. S., Alexandru, M., Racles, C., Nüesch, F. A., "Dielectric materials, design and realization," Proc. SPIE 9430, 94300A-1-94300A-10 (2015).

[7] Gallone, G., Galantini, F., Carpi, F., "Perspectives for new dielectric elastomers with improved electromechanical actuation performance: Composites versus blends," Polym. Int. 59(3), 400-406 (2010).

[8] Cazacu, M., Ignat, M., Racles, C., Cristea, M., Musteata, V., Ovezea, D., Lipcinski, D., "Well-defined siliconetitania composites with good performances in actuation and energy harvesting," J. Compos. Mater. 48(13), 1533-1545 (2014).

[9] Romasanta, L. J., Leret, P., Casaban, L., Hernández, M., de la Rubia, M. A., Fernández, J. F., Kenny, J. M., Lopez-Manchado, M. A., Verdejo, R., "Towards materials with enhanced electro-mechanical response: CaCu3Ti4O12-polydimethylsiloxane composites," J. Mater. Chem. 22, 24705-24712 (2012).

[10] Szabo, J. P., Hiltz, J. A., Cameron, C. G., Underhill, R. S., Massey, J., White, B., Leidner, J., "Elastomeric composites with high dielectric constant for use in Maxwell stress actuators," Proc. SPIE 5051, 180-190 (2003).

[11] Gharavi, N., Razzaghi Kashani, M., Moradi, A., "Electromechanical properties of silicone-PZT (lead-zirconatetitanate) composite," Proc. SPIE 7642, 764233-1-764233-7 (2010).

[12] Daugaard, E. A., Hassouneh, S. S., Kostrzewska, M., Bejenariu, A. G., Skov, A. L., "High-dielectric permittivity elastomers from well-dispersed expanded graphite in low concentrations," Proc. SPIE 8687, 868729-1-18687298 (2013).

[13] Opris, D. M., Molberg, M., Walder, C., Ko, Y. S., Fischer, B., Nüesch, F. A., "New silicone composites for dielectric elastomer actuator applications in competition with acrylic foil,” Adv. Funct. Mater. 21(18), 35313539 (2011).

[14] Molberg, M., Crespy, D., Rupper, P., Nüesch, F., Manson, J. A. E., Löwe, C., Opris, D. M., "High breakdown field dielectric elastomer actuators using encapsulated polyaniline as high dielectric constant filler," Adv. Funct. Mater. 20(19), 3280-3291 (2010).

[15] Chen, L. Z., Liu, C. H., Hu, C. H., Fan, S. S., "Electrothermal actuation based on carbon nanotube network in silicone elastomer," Appl. Phys. Lett. 92(26), 263104-1-263104-3 (2008).

[16] Carpi, F., Gallone, G., Galantini, F., De Rossi, D., "Silicone-poly(hexylthiophene) blends as elastomers with enhanced electromechanical transduction properties," Adv. Funct. Mater. 18(2), 235-241 (2008).

[17] Quinsaat, J. E. Q., Alexandru, M., Nüesch, F. A., Hofmann, H., Borgschulte, A., Opris, D. M., "Highly stretchable dielectric elastomer composites containing high volume fractions of silver nanoparticles," J. Mater. Chem. A 3(28), 14675-14685 (2015).

[18] Dünki, S. J., Tress, M., Kremer, F., Ko, S. Y., Nüesch, F. A., Varganici, C.-D., Racles, C., Opris, D. M., "Finetuning of the dielectric properties of polysiloxanes by chemical modification," RSC Adv. 5(62), 50054-50062 (2015). 
[19] Risse, S., Kussmaul, B., Krüger, H., Kofod, G., "Synergistic improvement of actuation properties with compatibilized high permittivity filler," Adv. Funct. Mater. 22(18), 3958-3962 (2012).

[20] Madsen, F. B., Dimitrov, I., Daugaard, A. E., Hvilsted, S., Skov, A. L., "Novel cross-linkers for PDMS networks for controlled and well distributed grafting of functionalities by click chemistry," Polym. Chem. 4, 1700-1707 (2013).

[21] Duenki, S. J., Ko, Y. S., Nueesch, F. A., Opris, D. M., "Self-repairable, high permittivity dielectric elastomers with large actuation strains at low electric fields," Adv. Funct. Mater. 25(16), 2467-2475 (2015).

[22] Opris, D. M., Dünki, S. J., Racles, C., Bele, A., Cazacu, M., "High permittivity polymers based on functionalized silicones," WO2015135086 (A1) (2015).

[23] Stepp, M., Achenbach, F., Koellnberger, A., "Vernetzbare Mischungen mit alpha-funktionellen Polysiloxanen," WO2015121261A1 (2014).

[24] Dubois, P., Coulembier, O., Raquez, J.-M., [Handbook of Ring-Opening Polymerization], John Wiley \& Sons, Weinheim 1-51 (2009).

[25] Rozga-Wijas, K., Chojnowski, J., Boileau, S., "Optically Active Dimethylsiloxane Copolymers with Nucleophilic Chiral Sulfur Groups Pendant to the Polysiloxane Chain,” J. Polym. Sci. Part A-Polymer Chem. 35(5), 879-888 (1997).

[26] Wright, P. V, Semlyen, J. A., "Equilibrium ring concentrations and the statistical conformations of polymer chains: Part 3. Substituent effects in polysiloxane systems," Polymer. 11(9), 462-471 (1970).

[27] Racles, C., Alexandru, M., Bele, A., Musteata, V. E., Cazacu, M., Opris, D. M., "Chemical modification of polysiloxanes with polar pendant groups by co-hydrosilylation," RSC Adv. 4(71), 37620-37628 (2014).

[28] Opris, D. M., unpublished results.

[29] Racles, C., Cazacu, M., Fischer, B., Opris, D. M., "Synthesis and characterization of silicones containing cyanopropyl groups and their use in dielectric elastomer actuators," Smart Mater. Struct. 22(10), 10 (2013).

[30] Racles, C., Bele, A., Dascalu, M., Musteata, V. E., Varganici, C. D., Ionita, D., Vlad, S., Cazacu, M., Dünki, S. J., et al., "Polar-nonpolar interconnected elastic networks with increased permittivity and high breakdown fields for dielectric elastomer transducers," RSC Adv. 5(72), 58428-58438 (2015).

[31] Dünki, S. J., Opris, D. M., unpublished results.

[32] Opris, D., Dascalu, M., Dünki, S., Quinsaat, J. E., Ko, Y. S., "Synthesis of silicone elastomers containing trifluoropropyl groups and their use in dielectric elastomer transducers," RSC Adv. 5, 104516-104523 (2015). 